\title{
Outcome of shunt operation on urinary incontinence in normal pressure hydrocephalus predicted by lumbar puncture
}

\author{
J AHLBERG, L NORLÉN,* C BLOMSTRAND, C WIKKELSÖ \\ From the Departments of Neurology and Urology, ${ }^{*}$ University of Göteborg, Göteborg, Sweden
}

SUMMARY Bladder function in four patients with normal pressure hydrocephalus, one with dementia of Alzheimer type and five patients with multi-infarct dementia was studied by history and urodynamic tests (cystometry and Bor's ice water test). The bladder hyperactivity could be temporarily improved by a lumbar puncture and removal of $50 \mathrm{ml}$ CSF and later abolished by a shunt operation in patients with normal pressure hydrocephalus while no changes occurred in the other patients. Urodynamic testing in connection with a lumbar puncture may be a reliable way to predict the outcome of a shunt operation in cases of normal pressure hydrocephalus.

The syndrome of gait disturbance, mental deterioration and urinary incontinence in conjunction with ventricular enlargement and normal cerebrospinal fluid (CSF) pressure is often referred to as normal pressure hydrocephalus. Urinary incontinence is usually the last symptom in the triad to appear but it is well known that each symptom may be found independently of the others. ${ }^{1}$ The incontinence, most often accompanied by frequency and urgency, is due to loss of voluntary supraspinal control of micturition (uninhibition) inducing hyperactivity and impairment of bladder sensation. In general, the prognosis in patients with this kind of bladder hyperactivity which can be caused by various brain lesions, is poor, owing to the fact that the majority of cases are caused by irreversible cerebral lesions. However, Hakim and Adams $^{2}$ showed that the three main symptoms of normal pressure hydrocephalus including incontinence could be relieved by a ventriculo-peritoneal shunt. Several authors ${ }^{2-4}$ have reported temporary improvement in gait pattern and mental function after lumbar puncture and the degree of improvement

Address for reprint requests: Dr Jarl Ahlberg, Department of Neurology, Sahlgren Hospital S-413 45 Göteborg, Sweden.

Received 3 June 1986.

Accepted 23 September 1986 was shown to correlate positively to the effect of a shunt operation. ${ }^{5}$

The present investigation aimed to evaluate if micturition symptoms in suspected normal pressure hydrocephalus could be temporarily improved by a lumbar puncture and thereby possibly predict a favourable outcome from a shunt operation.

\section{Material and methods}

Ten consecutive patients (seven men and three women) with a mean age of 67 years (range 53-72 years) admitted with suspected normal pressure hydrocephalus were included in the present study. Seven patients suffered from urinary incontinence, two had a history of urgency without incontinence and another patient had no micturition disturbances. Seven of the 10 patients suffered from gait disturbance and all had some degree of mental deterioration (table).

All patients were submitted to cranial computed tomography (CT), radionuclide cisternography ( ${ }^{99 \mathrm{~m}}$ Tc-DTPA) and EEG. Also, routine CSF, blood and urine examinations were performed to obtain a definitive diagnosis.

Further, a CSF tap-test according to Wikkelsö et $a l^{4}$ was performed on all patients on two consecutive days and at the same hour each day. On the first day 
the patients were tested psychometrically, the walking pattern was analysed and bladder function evaluated. On the second day a lumbar puncture was performed removing $50 \mathrm{ml}$ CSF 2-3 hours before the examinations from the first day were repeated. Bladder function was evaluated by history and urodynamic tests (cystometry and Bors ice water test).

Cystometry was performed according to the following procedure. After the patient had emptied the bladder two baby feeding tubes $(\mathrm{Ch} 8)$ were introduced into the bladder and the postvoid residual determined. Saline at room temperature was infused into the bladder at a rate of approximately $40 \mathrm{ml} / \mathrm{min}$ via one of the tubes. Via the other tube bladder pressure was continuously recorded on an ink recorder (Mingograph 81, Siemens Elema) via a pressure transducer (EMT 34, Siemens Elema) and an amplifier. Simultaneously, the rectal pressure was recorded and was electronically subtracted from the bladder pressure to obtain the "true" intravesical pressure. Involuntary detrusor contractions during filling of an amplitude of more than $15 \mathrm{~cm} \mathrm{H}_{2} \mathrm{O}$ and/or cystometric capacity of less than $225 \mathrm{ml}$ was taken as a sign of detrusor instability.

Bors ice water test was performed in the following manner. Sterile icecold water $(100 \mathrm{ml})$ was rapidly introduced into the bladder via a syringe and the intravesical pressure was simultaneously recorded. If the bladder reacted with contraction the test was regarded as positive and if there was no bladder contraction the test was regarded as negative. The test is positive in uninhibited bladders and marked detrusor instability.

Patients with clinical and laboratory signs of normal pressure hydrocephalus were operated upon by a ventriculo-peritoneal shunt. Three months after surgery these patients were reinvestigated with psychometric tests, walking analysis and evaluation of bladder function.

\section{Results}

Of the 10 patients admitted with suspected normal pressure hydrocephalus the diagnosis was confirmed in four patients (patients 1-4); three of them had had previous subarachnoidal haemorrhage and one a history of severe head trauma. One patient was found to have dementia of Alzheimer type (DAT) (patient 5) and five patients had multi-infarct dementia (MID) (patients 6-10), one in combination with chronic alcohol abuse (patient 6).

All four patients diagnosed as suffering from normal pressure hydrocephalus complained of urge incontinence. On urodynamic testing before the $C S F$ tap-test three patients (patients 1-3) had detrusor instability and one had a stable bladder. However, all four patients had positive Bors ice water test. After CSF tap-test detrusor instability was abolished in two patients (patient 1 and 3) (fig) and reduced in one patient (patient 2) but Bors' ice water test remained positive in these three patients. However, in one patient Bors' ice water test changed from positive to negative (patient 4).

In the six patients diagnosed as having DAT or MID all but one (patient 5) had bladder symptoms (urge and/or incontinence). Three of the patients had융 normal urodynamics (patients 5,6 and 9) and three? had detrusor instability (patients 7,8 and 19) in one 2 of whom Bors' ice water test was positive (patient 8) After the CSF tap-test the urodynamic testing waso unaltered in these three patients.

Bladder function was again evaluated in the four patients with normal pressure hydrocephalus 3 응 months after operation with a ventriculo-peritoneal shunt. All four had normal micturition pattern on urodynamic testing and Bors' ice water test was negative. Three patients had no signs of instability on cystometry (table) while one patient had detrusor instability; however, this was further reduced

Table Details of the 10 patients

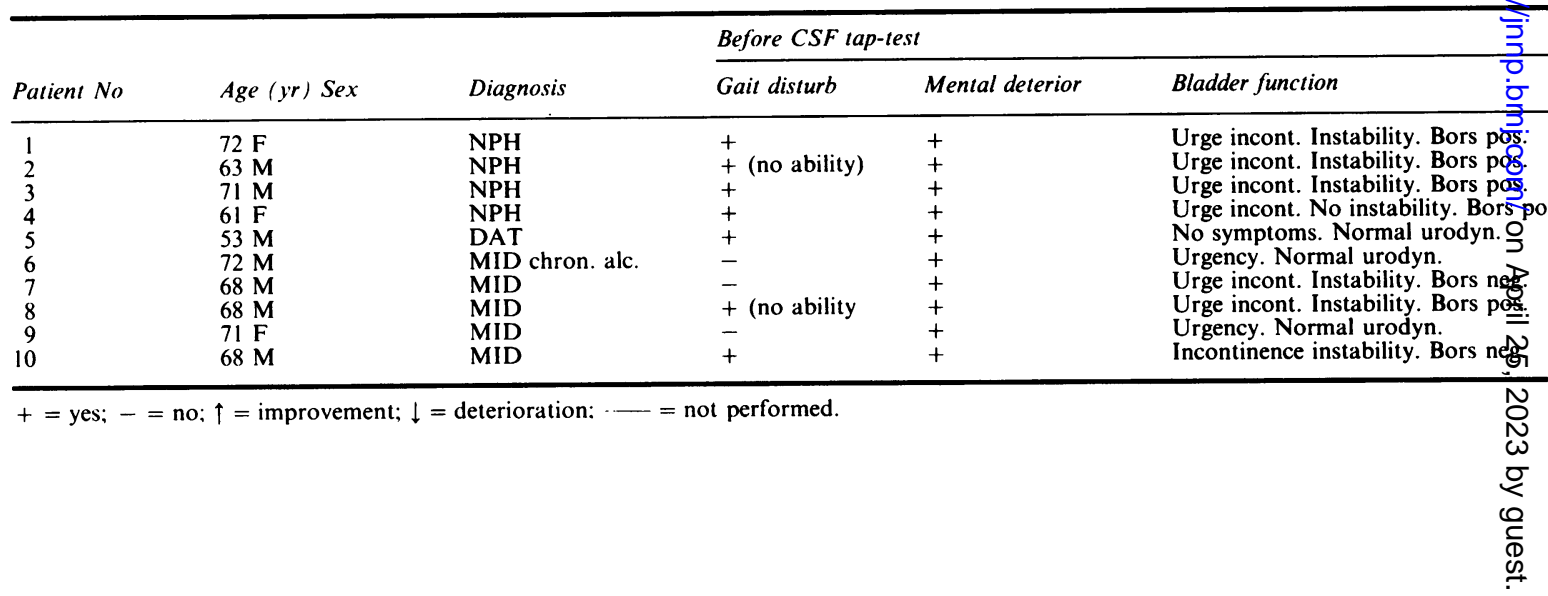




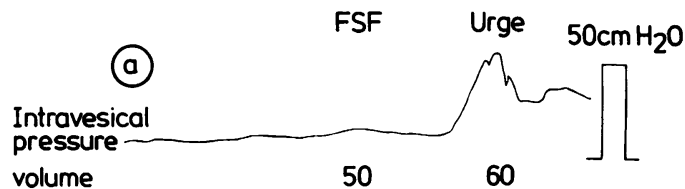

(b)

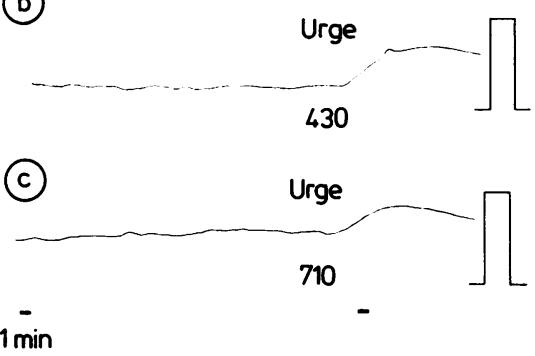

Fig 72-year-old woman with normal pressure hydrocephalus (patient 1). (A) Before CSF tap-test cystometry showed first sensation of filling at $50 \mathrm{ml}$ and suddenly involuntary detrusor contraction and leakage combined with urge at $60 \mathrm{ml}$. (B) After CSF tap-test patient experiences urge to void at $430 \mathrm{ml}$ and empties with voluntary detrusor contraction. (C) After shunt operation patient experiences urge to void at $710 \mathrm{ml}$ and empties with voluntary detrusor contraction.

compared with before and after CSF tap-test.

Bladder functions before and after CSF tap-test and after operation are summarised in the table. For comparison results of CSF tap-test on gait and mental status are also given in the table.

\section{Discussion}

Micturition and storage of urine is normally controlled by the CNS through mechanisms which are complicated and not fully understood. ${ }^{6}$ Neurogenic bladder disturbances may occur after various defined brain lesions such as tumour, haemorrhage or contusion and is characterised by uninhibition of micturition, that is, the patient is not able to suppress micturition and is incontinent. From animal experiments and clinical observations it appears that the supraspinal control of the bladder can be mainly referred to three areas, the frontal cerebral cortex, hypothalamic structures and the brain stem. ${ }^{67}$ However, disturbances of bladder function may also occur in conditions with more generalised brain disorders such as degenerative CNS disorders, multi-infarct dementia and hydrocephalus. ${ }^{6}$

In normal pressure hydrocephalus bladder disturbance has been considered to be a frontal lobe incontinence, because the patients are indifferent regarding where and when to urinate. ${ }^{1}$ None of the patients in the present study showed this form of incontinence and none of them had such severe mental impairment or gait disturbance that the incontinence could be thus explained. Further, direct changes in the bladder functions could be noted after removal of CSF. These observations support the notion that bladder symptoms in normal pressure hydrocephalus are rather due to injury in regions important for micturition. When the improvement in the urodynamic tests was compared with the improvement in gait pattern and psychometric test score no direct parallel between the degree of improvement in the different parameters could be found. This indicates that bladder impairment and mental deterioration are two separate entities in the triad of normal pressure hydrocephalus, which is also supported by the fact that the symptoms can be found independently of each other. Still the possibility remains that the mental deterioration commonly including impaired wakefulness in combination with the gait disturbances increases the risk of incontinence in patients with urgency.

In the present study bladder hyperactivity was confirmed on urodynamic evaluation in all four patients with normal pressure hydrocephalus. The

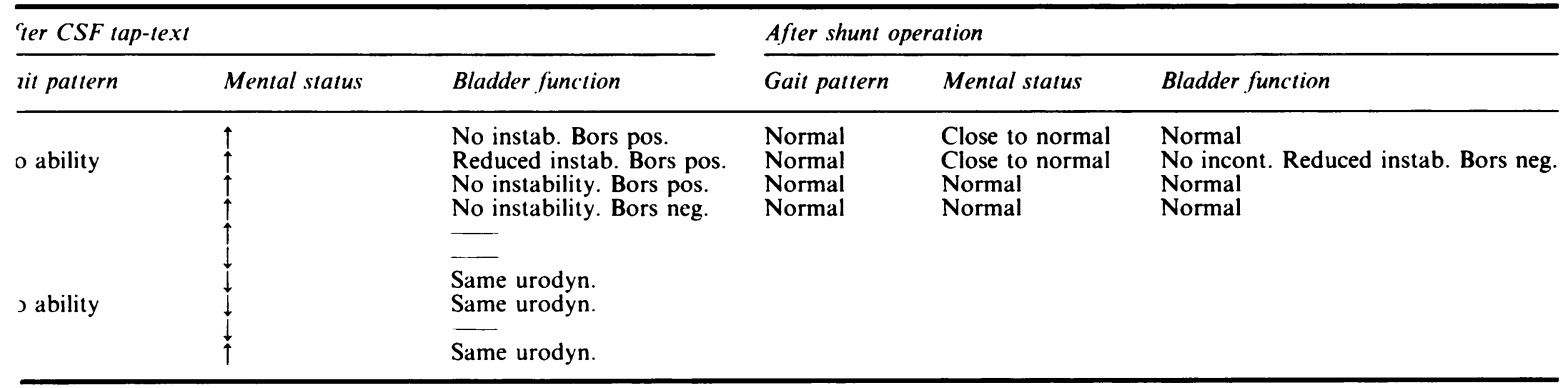


hyperactivity in cerebral lesions is due to uninhibition causing incontinence which is part of the syndrome in normal pressure hydrocephalus. As previously noted $^{124}$ gait disturbance and mental deterioration could be temporarily improved after CSF tapping. The bladder hyperactivity was decreased in all four patients with normal pressure hydrocephalus after the lumbar puncture together with an improvement in gait pattern and mental status. Thus, urodynamic evaluation seems to be a useful method in evaluation of bladder function in normal pressure hydrocephalus before and after the CSF tap-test.

Recently it was shown ${ }^{5}$ that the degree of improvement in gait pattern and mental status after lumbar puncture correlated with the effect of a ventriculo-peritoneal shunt operation. In the present study bladder symptoms were totally abolished 3 months after the operation and the urodynamic pattern was normalised in three patients and substantially improved in one of the four patients with normal pressure hydrocephalus.

Of the other six patients in the present study three with MID and incontinence had abnormal bladder activity on urodynamic testing. After lumbar puncture there was no change in the urodynamic tests.

In summary, bladder disturbances in normal pressure hydrocephalus were temporarily improved after lumbar puncture and permanently abolished or substantially improved after ventriculo-peritoneal shunt operation. Thus, lumbar puncture and urodynamic testing may be a reliable way to predict the outcome of shunt operation in patients with urinary incontinence due to normal pressure hydrocephalus.

This study was supported by grants from the Edith Jacobson's foundation and from the Swedish Medical Research Council (project No. 066602).

\section{References}

1 Fisher CM. Hydrocephalus as a cause of disturbances of gait in the elderly. Neurology 1982;32:1358-63.

2 Hakim S, Adams RD. The special clinical problem of symptomatic hydrocephalus with normal cerebrospinal fluid pressure. Observations on cerebrospinal fluid hydrodynamics. J Neurol Sci 1965;2:307-27.

3 Fisher CM. The clinical picture in occult hydrocephalus. Clin Neurosurg 1977;24:270-84.

4 Wikkelsö C, Andersson H, Blomstrand C, Lindqvist G. The clinical effect of lumbar puncture in normal pressure hydrocephalus. Description of a diagnostic CSF tap-test. J Neurol Neurosurg Psychiatry 1982;45:64-9.

5 Wikkelsö C, Andersson H, Blomstrand C, Lindqvist G, Svendsen P. Normal pressure hydrocephalus. Predictive value of the cerebrospinal fluid tap-test. Acta Neurol Scand 1986 (in press.)

6 Carlsson CA. The supraspinal control of the urinary bladder. Acta pharmacol et toxicol 1978;43:2963.

7 Gerstenberg TC, Gjerris F, Soelberg P, Sörensen and Hald T. Detrusor hyperreflexi and detrusor sphincter incoordination and conductance to cerebrospinal fluid outflow in normal pressure hydrocephalus. Acta Neurol Scand 1982;65,Suppl 90:296-7. 